\title{
FUNDAMENTAL THEOREMS OF SUMMABILITY THEORY FOR A NEW TYPE OF SUBSEQUENCES OF DOUBLE SEQUENCES
}

\author{
Raluca Dumitru And Jose A. Franco
}

\begin{abstract}
In 2000, the notion of a subsequence of a double sequence was introduced [3]. Using this definition, a multidimensional analogue to a result from $\mathrm{H}$. Steinhaus, that states that for any regular matrix $A$ there exists a sequence of zeros and ones that is not $A$-summable, was proved. Additionally, an analogue of a result of R. C. Buck that states that a sequence $x$ is convergent if and only if there exists a regular matrix $A$ that sums every subsequence of $x$ was presented. However, this definition imposes a restrictive condition on the entries of the double sequence that can be considered for the subsequence. In this article, we introduce a less restrictive new definition of a subsequence. We denote them by $\beta$-subsequences of a double sequence and show that analogues to these two fundamental theorems of summability still hold for these new subsequences.
\end{abstract}

Mathematics subject classification (2010): 40B05, 40A05.

Keywords and phrases: Subsequences of a double sequence, $\beta$-sections, convergence, divergence, limit points, Pringsheim.

\section{REFERENCES}

[1] Buck, R. C., A note on subsequences, Bull. Amer. Math. Soc. 49, (1943), 898-899.

[2] Hamilton, H. J., Transformations of multiple sequences, Duke Math. J. 2, 1 (1936), 29-60.

[3] Patterson, R. F., Analogues of some fundamental theorems of summability theory, Int. J. Math. Math. Sci. 23, 1 (2000), 1-9.

[4] Patterson, R. F., AND RhoAdes, B. E., Summability of $\lambda$-rearrangements for double sequences, Analysis (Munich) 24, 3 (2004), 213-225.

[5] Pringsheim, A., Zur Theorie der zweifach unendlichen Zahlenfolgen, Math. Ann. 53, 3 (1900), 289-321.

[6] Robison, G. M., Divergent double sequences and series, Trans. Amer. Math. Soc. 28, 1 (1926), $50-73$. 